\title{
Symptomatic and silent atrial fibrillation recurrences after pulmonary vein isolation ablation - usefulness of prolonged 7-day Holter recordings. One-center observation
}

\author{
Marcin Wita ${ }^{1}$, Andrzej Hoffmann ${ }^{1}$, Krzysztof Szydło ${ }^{1}$, Seweryn Nowak ${ }^{1}$, Urszula Uchwat ${ }^{2}$, Katarzyna Mizia-Stec $^{1}$ \\ ${ }^{1}$ First Department of Cardiology, School of Medicine in Katowice, Medical University of Silesia, Katowice, Poland \\ ${ }^{2}$ Department of Neurology with the Stroke Subdepartment, Upper Silesian Medical Centre, Katowice, Poland
}

Adv Interv Cardiol 2019; 15, 2 (56): 255-257

DOI: https://doi.org/10.5114/aic.2019.83654

\section{Introduction}

Atrial fibrillation (AF), especially its paroxysmal form (paroxysmal atrial fibrillation - PAF), is still the most common arrhythmia observed in cardiological practice. It is well known that some diseases may predispose patients to PAF occurrence - such as hypertension, coronary artery disease, valvular heart disease, congestive heart failure, pericarditis, thyrotoxic heart disease, sinus node disease, but also hypokalemia and alcohol overconsumption. However, especially in younger individuals, arrhythmia may occur without any evidenced disease or reason. It should be emphasized that up to $30-40 \%$ of episodes are completely asymptomatic (silent atrial fibrillation), and there are some data indicating that this form of PAF is especially often observed in patients after ablation procedures. Cardiac denervation caused by ablation is probably responsible for this phenomenon. These observations seem to be very important, because catheter ablation, according to the latest European Society of Cardiology (ESC) guidelines, has become a preferable and commonly used method of treatment, especially in young and highly symptomatic patients with drug refractory arrhythmia [1]. Some different ablation strategies were proposed, but pulmonary vein isolation (PVI) is a cornerstone of AF ablation, nowadays. Unfortunately, reconnections of PV-atrium junctions and/or non-pulmonary localization of AF trigger may be responsible for limitation of the ablation success rate. Absolute effectiveness of PVI in patients with PAF is still not well known because studies based on the follow-up using only the clinical symptoms have a limited value [2, 3]. More precise data might be obtained with the use of long-term continuous ECG recordings, e.g. several-day Holter recordings or tele-
ECG methods. Therefore, the purpose of this study was to compare results obtained from 1-day and continuous 7-day Holter recordings performed in patients with PAF in regard to relapses of arrhythmia after PVI.

\section{Aim}

We estimated the presence of PAF and the correlation between symptoms and arrhythmia recurrences.

\section{Material and methods}

We prospectively observed 102 highly symptomatic patients with drug-refractory PAF (at least 2-3 antiarrhythmic agents were used in history) or with intolerance of antiarrhythmic drugs, qualified for PVI in the years 2014-2016. All of these patients were treated by radiofrequency point-by-point circumferential pulmonary vein ablation. The procedure was performed using an electroanatomical 3D system: CARTO (Biosense Webster) or EnSite (St. Jude Medical). Rotational angiography at the beginning of the procedure was performed in all patients. Ablation was performed with an open-irrigated tip catheter in all patients. Circular decapolar diagnostic catheters were used to document the isolation of pulmonary veins in every patient. If the procedure was performed during atrial fibrillation, after isolation of all veins, sinus rhythm was restored (pharmacologically or by electrical cardioversion). At the end of the ablation entrance and exit block were documented in all pulmonary veins. Each patient was followed up every 3 months after the ablation, for a minimum of 1 year. Holter recordings were performed 6-9 months (median: 7 months) after the procedure (continuous 7-days recording, H7) using the Reynolds Pathfinder 700 system. An episode of PAF 
was diagnosed when the duration was $>30 \mathrm{~s}$. Two PAFs were regarded as one episode, when the duration of the sinus rhythm between them was $<30 \mathrm{~s}$. We analyzed the presence of PAF on the first day of Holter monitoring (H7F) and in the rest of the recording $\left(\mathrm{H}_{2}\right.$ 7 7$)$. When a few episodes were observed in the recording, the first one was the end point episode. The duration of all episodes was also calculated. Our patients were asked to maintain a log of symptoms suggestive of $A F$, then the individual AF episode was classified as either symptomatic or asymptomatic.

\section{Statistical analysis}

The continuous variables are presented as the mean \pm standard deviation, the categorical variables as the number or percentage of subjects. Differences were assessed using Student's $t$ test, while non-normal data were compared using the Mann-Whitney $U$ test. The normality of distribution was verified with the Shapiro-Wilk test. The categorical variables were compared using the $\chi^{2}$ test. A $p$-value of $<0.05$ was considered to be statistically significant. All statistical analyses were conducted using Statistica 12.

\section{Results}

Eight patients had recurrence of AF confirmed with 12-lead standard ECG recording during the follow-up - all episodes were symptomatic. Therefore, further observation with $\mathrm{H} 7$ recordings was performed on the group of 94 patients (64 males, age $55 \pm 10$ years, $\mathrm{CHA}_{2} \mathrm{DS}_{2}$-VASc score $1.4 \pm 1.1$, median $=1$ ). Fifty-eight patients (41 males, age $55 \pm 11$ years $\mathrm{CHA}_{2} \mathrm{DS}_{2}$-VASc) $1.4 \pm 1.5$ median $=1$, $\beta$-blocker in 48 , amiodarone in 4 patients) were asymptomatic after PVI with no relapse of PAF in standard ECG as well as in $\mathrm{H} 7$ recordings. Forty patients were still symp- tomatic, but recurrences of PAF were documented with $\mathrm{H} 7$ recordings only in 36 patients (23 males, age $56 \pm 9$ years, $\mathrm{CHA}_{2} \mathrm{DS}_{2}$-VASc $1.4 \pm 0.7$ median $=1$, $\beta$-blocker in 32 and amiodarone in 5 patients), and in 4 subjects single premature contractions (atrial and/or ventricular) were observed during reported symptoms. Symptomatic and silent patients did not differ in age, gender or treatment after PVI. All patients had left ventricle ejection fraction $>50 \%$ in echocardiographic examination with no evidence of valvular heart disease, and none had coronary artery disease.

The occurrence of symptomatic and silent PAF episodes is given in Table I. Silent PAF was found in 4 patients on the first day of prolonged ECG recording and in 5 patients during the next days. Symptomatic episodes were observed in 16 and 11 patients, respectively. Prolongation of ECG recording to 7 days improved PAF detection by $80 \%$ in the entire population, and by $69 \%$ and $125 \%$ in symptomatic and silent patients, respectively. Total time of silent PAF was significantly shorter both in $\mathrm{H} 7 \mathrm{~F}$ and H2_7. The precise data of PAF recurrences are presented in Table II. Five (55.5\%) from 9 silent patients had 2 or more points in the $\mathrm{CHA}_{2} \mathrm{DS}_{2}$-VASc score. None of them had 1 point.

\section{Discussion}

The main finding of this study is that prolongation of ECG recording from standard 24-hour (typical Holter recording) up to 7 days may improve detection of PAF by as much as 2-fold. We also found 9 cases of silent PAF in complete asymptomatic patients. It should be emphasized that silent PAF episodes were shorter that symptomatic episodes, but all of them had duration of at least $10 \mathrm{~min}$. No evidence of PAF in asymptomatic patients allowed for termination of antithrombotic treatment

Table I. Atrial fibrillation episodes in $\mathrm{H} 7$ recordings

\begin{tabular}{|c|c|c|c|}
\hline Parameter & 1 day (H7F) & $\begin{array}{l}\text { Only during 2-6 days } \\
\qquad\left(\mathrm{H} 2 \_7\right)\end{array}$ & 7 days $(\mathrm{H} 7)$ \\
\hline Symptomatic & $16(44.4 \%)$ & $11(30.6 \%)$ & $27(75 \%)$ \\
\hline Silent & $4(11.1 \%)$ & $5(13.9 \%)$ & $9(25 \%)$ \\
\hline Total number of PAF episodes & $20(55.5 \%)$ & $16(44.5 \%)$ & $36(100 \%)$ \\
\hline Total time of silent PAF & $1.8 \pm 2.4 \mathrm{~h}$ & $12.1 \pm 5.3 \mathrm{~h}$ & $p=0.001$ \\
\hline Total time of symptomatic PAF & $\begin{array}{c}7.1 \pm 9 h \\
p=0.001^{\star}\end{array}$ & $\begin{array}{c}59 \pm 12 \mathrm{~h} \\
p=0.001^{*}\end{array}$ & $p=0.001$ \\
\hline Symptomatic during PAC/PVC & 0 & 4 & 4 \\
\hline
\end{tabular}

$P A C$ - premature atrial contraction, $P V C$ - premature ventricular contraction. ${ }^{*} P$-value for time of PAF silent vs. symptomatic.

Table II. The PAF recurrences

\begin{tabular}{|c|c|c|c|c|c|c|c|}
\hline Variable & Day 1 & Day 2 & Day 3 & Day 4 & Day 5 & Day 6 & Day 7 \\
\hline AF patients & 20 & 5 & 1 & 3 & 2 & 3 & 2 \\
\hline Symptomatic/silent & $15 / 4$ & $5 / 0$ & $1 / 0$ & $2 / 1$ & $0 / 2$ & $2 / 1$ & $1 / 1$ \\
\hline
\end{tabular}


in 8 from 14 patients with a $\mathrm{CHA}_{2} \mathrm{DS}_{2}$-VASc score $=1$. Discontinuation of the antithrombotic treatment in $5 \mathrm{si}$ lent patients with a $\mathrm{CHA}_{2} \mathrm{DS}_{2}$-VASc score of 2 or more was impossible.

Prolonged ECG recordings were performed after 6-9 months after the $\mathrm{PVI}$ procedure. It is proven that AF episodes observed during the first months after the different types of ablation should not be correlated with lack of the effectiveness of the procedure-blanking period [4]. The long-term effectiveness of PVI procedures is one of the most important end-points of many studies. Results of 24-hour Holter recordings and symptoms of patients were used in many of them. Scherr et al. [5] analyzed long-term effectiveness of PVI (one or repeated procedure) in patients with persistent AF. They found that arrhythmia-free survival rates after the last procedure were $89 \%, 79 \%$, and 63 , at 1,2 , and 5 years, respectively. In our study the effectiveness after the first procedure was 55\% during 6-9-month follow-up. Some of our patients were qualified for the next ablation, but it was not our point of interest in this study. Moreover, no data on silent AF were given by Scherr et al. A meta-analysis of 13 different studies (1774 patients) [6] showed that the 12 -month success rate of a single ablation procedure was $78 \%$. However, different types of ablation procedures were used in these studies, and there were data on silent AF as well.

The usefulness of prolonged ECG recordings was reported in 2010 by Dagres et al. [7]. They analyzed data of 250 patients after AF ablation who underwent 7-day Holter ECG at 6 months after the catheter ablation. The recurrence of AF was documented in $30 \%$ of patients, but only $59 \%$ of them had arrhythmia in the first day of ECG monitoring. Similar data are presented in our study, but we could not discuss silent AF; the percentage of this form of AF was not given by Dagres et al. The first data on silent AF can be found in the paper by Hindricks et al. [8]. They prospectively studied a group of 114 patients who underwent circumferential lesions around the pulmonary veins - the older method of AF ablation. They assessed the occurrence of AF episodes 3, 6 and 12 months after ablation using 7-day Holter recordings. The authors found that after ablation the number of asymptomatic AF episodes increased from $5 \%$ to $36-38 \%$. In our study silent AF episodes occurred in $25 \%$ of the study group. Interesting results were presented recently by Nielsen et al. [9]. They also used 7-day Holter recording to assess the effectiveness of antiarrhythmics therapy and AF ablation during 5-year follow-up. They analyzed 245 patients in the ablation arm, but only 227 had 7 -day recording; moreover, 25 patients underwent a second ablation. They found $91 \%$ of patients free of any arrhythmia; they found the silent form of AF only in 6 patients.

Others studies have reported different proportions between silent and symptomatic forms of $A F$, and different levels of success of the ablation procedure, as well. Further studies are necessary to assess the recurrences of silent AF in patients after PVI or any other ablation method, especially in those with a $\mathrm{CHA}_{2} \mathrm{DS}_{2}$-VASc score $=1$. Our study suggests that silent PAF episodes were shorter, which might explain the lack of symptoms in these patients.

\section{Conclusions}

Seven-days Holter monitoring is a helpful tool to assess the efficacy of pulmonary vein isolation in patients with PAF. Silent episodes of PAF are not very rare in that population.

\section{Conflict of interest}

The authors declare no conflict of interest.

\section{References}

1. Kirchhof P, Benussi S, Kotecha D, et al. 2016 ESC Guidelines for the management of atrial fibrillation developed in collaboration with EACTS The Task Force for the management of atrial fibrillation of the European Society of Cardiology (ESC) Developed with the special contribution of the European Heart Rhythm Association (EHRA) of the ESC Endorsed by the European Stroke Organisation (ESO). Eur Heart J 2016; 37: 2893-962.

2. Oral H, Veerareddy S, Good E, et al. Prevalence of asymptomatic recurrences of atrial fibrillation after successful radiofrequency ablation. J Cardiovasc Electrophysiol 2004; 15: 920-4.

3. Marrouche NF, Natale A. Atrial fibrillation ablation: role of patient enrollment and follow-up in clinical results. J Am Coll Cardiol 2003; 41: 1232-3.

4. Joshi S, Choi A, Kamath G, et al. Prevalence, predictors, and prognosis of atrial fibrillation early after pulmonary vein isolation: findings from 3 months of continuous automatic ECG loop recordings. J Cardiovasc Electrophysiol 2009; 20: 1089-94.

5. Scherr D, Khairy P, Miyazaki S, et al. Five-year outcome of catheter ablation of persistent atrial fibrillation using termination of atrial fibrillation as a procedural endpoint. Circ Arrhythm Electrophysiol 2015; 8: 18-24.

6. Kis Z, Muka T, Franco O, et al. The short and long-term efficacy of pulmonary vein isolation as a sole treatment strategy for paroxysmal atrial fibrillation: a systematic review and meta-analysis. Curr Cardiol Rev 2017; 13: 199-208.

7. Dagres N, Kottkamp H, Piorkowski C, et al. Influence of the duration of Holter monitoring on the detection of arrhythmia recurrences after catheter ablation of atrial fibrillation: implications for patient follow-up. Int J Cardiol 2010; 139: 305-6.

8. Hindricks G, Piorkowski C, Tanner $\mathrm{H}$, et al. Perception of atrial fibrillation before and after radiofrequency catheter ablation. Relevance of asymptomatic arrhythmia recurrence. Circulation 2005; 112: 307-13.

9. Nielsen J, Johannessen A, Raatikainen P, et al. Long-term efficacy of catheter ablation as first-line therapy for paroxysmal atrial fibrillation: 5-year outcome in a randomised clinical trial. Heart 2017; 103: 368-76. 